\title{
Del salón al aula virtual: Las dificultades tecnológicas, económicas y de salud mental que afrontan los universitarios para el desarrollo de la educación remota en el marco de la pandemia del COVID-19
}

\section{From the classroom to the virtual classroom: The technological, economic and mental health challenges facing university students in developing remote education in the context of the COVID-19 pandemic}

http://dx.doi.org/10.17981/cultedusoc.12.2.2021.12

Recibido: 24 de noviembre de 2020 Aceptado: 16 de febrero de 2021 Publicado: 1 de julio de 2021.

Andrea Katherine García-González (i)

Corporación Unificada Nacional de Educación Superior CUN. Bogotá, D.C. (Colombia)

andrea_garcia@cun.edu.co

Danilo Esteban Rodríguez-Zapata

Corporación Unificada Nacional de Educación Superior CUN. Bogotá, D.C. (Colombia)

danilo_rodriguez@cun.edu.co

Para citar este artículo:

García-González, A. y Rodríguez-Zapata, D. (2021). Del salón al aula virtual: Las dificultades tecnológicas, económicas y de salud mental que afrontan los universitarios para el desarrollo de la educación remota en el marco de la pandemia del COVID-19. Cultura, Educación y Sociedad, 12(2), 205-222. DOI: http://dx.doi.org/10.17981/cultedusoc.12.2.2021.12

\section{Resumen}

En este artículo se abordan los efectos económicos, sociales y educativos provocados por los confinamientos obligatorios en respuesta al problema de salud pública mundial que representa la pandemia del COVID-19. Esto, en el marco del cierre de las instituciones educativas en su modalidad presencial y el paso imperativo a la modalidad remota o virtual. Así pues, en este documento se pretenden comprender las dificultades en términos tecnológicos, de salud mental, y de recursos económicos que enfrentan estudiantes de una Institución de Educación Superior, con cobertura nacional y pertenecientes a la clase media colombiana. Por lo cual, se realizó una indagación de tipo descriptiva y mixta a partir de grupos focales, entrevistas abiertas y encuestas a estudiantes, docentes y directivos. Se encontró que el principal obstáculo en lo tecnológico es la conectividad a través de una red estable y de alta velocidad. Estas falencias se profundizaron en la ruralidad. En relación con la salud mental, se reconoció la presencia de distintos estresores en los estudiantes que han sido identificados en la crisis actual y en investigaciones previas sobre otros contextos de desastres sanitarios. Y, por último, se evidenció la preocupación generalizada de los estudiantes por los impactos económicos de la pandemia y las posibilidades de continuar sus procesos de formación académica.

Palabras clave: Universitarios; educación remota; virtualidad; COVID

\section{Abstract}

This article addresses the economic, social and educational effects of mandatory lockdowns in response to the global public health problem caused by the COVID19 pandemic. This, in the context of the closure of educational institutions in its face-to-face modality and the imperative passage to the remote or virtual modality. Thus, this paper seeks to understand the difficulties in terms of technology, mental health, and economic resources faced by students of a Higher Education Institution, with national coverage and belonging to the middle class of the country. Therefore, a descriptive and mixed type of research was carried out through focus groups, open interviews and surveys to students, teachers and directors. It was found that the main technological obstacle is connectivity through a stable and high-speed network. These deficiencies were more severe in rural areas. In relation to mental health, the presence of different stressors in students that have been identified in the current crisis and in previous research on other health disaster contexts was recognized. And, finally, there was evidence of widespread concern among students about the economic impacts of the pandemic and the possibilities of continuing their academic training processes.

Keywords: University students; remote education; virtuality; COVID 


\section{INTRODUCCIÓN}

Según la Comisión Económica para América Latina y el Caribe (CEPAL, 2020), más de 190 países en el mundo han sufrido interrupciones educativas en el transcurso del 2020 debido a la necesidad de mantener el distanciamiento social para evitar la propagación del COVID-19, y habría más de 1200 millones de estudiantes afectados, de los cuales más de 160 millones corresponden a Latinoamérica. El sistema educativo de Colombia no ha sido ajeno a esta situación. En los diferentes niveles, desde guarderías, preescolares, hasta colegios, universidades e instituciones de formación para el trabajo, se vieron obligados a cerrar sus puertas, afectando con esto a estudiantes, docentes, personal administrativo, directivo y de apoyo, incluso a padres de familia. Con el aislamiento repentino los centros educativos debieron adaptarse prontamente a la enseñanza remota. Sin embargo, este redireccionamiento de los procesos tradicionales de enseñanza y aprendizaje, que en la mayoría del país y sus instituciones tenían un carácter estrictamente presencial, desconoció la realidad de la mayoría de los estudiantes, de sus familias y de sus contextos.

Es posible mencionar tres grandes dificultades para el desarrollo de la modalidad de educación remota o virtual en Colombia. En primer lugar, la insatisfacción de necesidades básicas y el acceso a recursos y servicios, es de notar el alto porcentaje de personas clasificadas como pobres en el país, que según el Departamento Aministrativo Nacional de Estadística (DANE) para 2020 se trató del 42.5\% de los colombianos. Esta cifra empieza a develar las complejas limitaciones para la aplicación de una educación remota de calidad en un entorno donde una cuarta parte de la población tiene dificultades para alimentarse tres veces al día, vivir en un espacio fijo y salubre, entre otras cosas (DANE, 2021). Así pues, los escenarios de educación se están trasladando a espacios que no garantizan las condiciones materiales de existencia mínimas para una vida digna y que, por supuesto, no favorecen los procesos de aprendizaje.

En segundo lugar, se encuentra la falta de acceso a dispositivos tecnológicos y conectividad, como elementos esenciales para el desarrollo de las clases remotas. Sobre esto, basta conocer la tasa de hogares que posee un computador portatil (28.8\% para 2018) (DANE, 2019), un computador de escritorio (20.6\% para 2018) (DANE, 2019), o una tableta (10.9\% para 2018) (DANE, 2019) y, el porcentaje de hogares que cuenta con conexión a internet (52.7\% para 2018) (DANE, 2019). Estos datos nacionales denotan la carencia de los recursos tecnológicos mínimos indispensables para desarrollar esta modalidad de educación. A esta información se debe añadir el desconocimiento por parte de los diferentes actores del uso adecuado de estos elementos en un ambiente de formación. Esta situación es particularmente preocupante en las clases más vulnerables y los sectores rurales apartados, para quienes la virtualidad no es una opción y la deserción escolar se convierte en la alternativa principal. Esto preocupa al gobierno (Ministerio de Educación Nacional, MEN, 2020), a las familias y en general a la sociedad que advierte los impactos negativos que esto puede conllevar al futuro capital humano capacitado del país y, a largo plazo, al crecimiento económico nacional.

Y, en tercer lugar, las afectaciones psicológicas (e incluso físicas) profundizadas de manera alarmante a causa de la pandemia tales como, la violencia intrafamiliar. Según 
datos que la Fiscalía General de la Nación comunicó al periódico El Tiempo "cada día hay 249 denuncias por violencia intrafamiliar en Colombia" (Amat, 2020). De manera que, para varias familias en el país el confinamiento obligatorio los envío a una espiral de violencia, impidiendo que se puedan alejar de los agresores que pertenecen, en estos casos, al mismo núcleo familiar. En este punto, tampoco se puede desconocer la presencia que tienen grupos armados ilegales en los territorios y sus dinámicas que minimizan las posibilidades de acceso a la educación (Palacios, 2020). Dichas organizaciones delincuenciales mantienen el control sobre regiones, rurales y urbanas, abandonadas por el Estado, y coartan la posibilidad de los individuos de insertarse en procesos de formación.

Aunado a esto, se debe contemplar que, respecto al resto de países de Latinoamérica, Colombia está entre aquellos que ha presentado mayor movilización interna estudiantil debido al cierre de las aulas (Valdivieso, Burbano y Burbano, 2020). Estos desplazamientos se han dado principalmente desde el centro del país, particularmente de Bogotá, hacia las demás regiones periféricas, así como desde las cabeceras departamentales hacia los municipios y veredas. Estos movimientos se pueden explicar porque la educación, como muchos otros servicios, se encuentra centralizada en los ejes económicos y políticos (Radinger, Echazarra, Guerrero y Valenzuela, 2018). Así pues, cuando cesaron las actividades educativas presenciales, la mayoría de los estudiantes provenientes de otras partes del país debieron migrar a sus lugares de origen, principalmente por la imposibilidad de sostener su estadía económicamente. Dicho esto, se está, primordialmente, ante un problema de cobertura.

Según la Organización de las Naciones Unidas para la Educación, la Ciencia y la Cultura y el Instituto Internacional para la Educación Superior en América Latina y el Caribe (UNESCO/IESALC, 2020) respecto al contexto internacional, las tasas nacionales de cobertura no superan el 50\%. Con esta problemática de fondo se puede vislumbrar como los jóvenes que se habían desplazado para desarrollar sus estudios deberán volver a sus hogares que, en muchas ocasiones, no les ofrecen las condiciones necesarias para formarse remotamente. Es así como los inesperados cambios estructurales en el modelo educativo que debieron ejecutarse de manera expedita, ante la necesidad de migrar toda actividad a la virtualidad, reflejaron, así como en muchos otros campos, las profundas desigualdades y problemas que la presencialidad y la desconexión habían ocultado.

Ante todas estas dificultades se proyecta que la pandemia agudice las brechas de aprendizaje a nivel nacional e incremente las discrepancias en distintos contextos a la vez que genere nuevas brechas. Esto en la medida que los territorios alejados, así como los grupos poblacionales más vulnerables carecerán de acceso a la educación, o de acceso a un servicio de calidad que garantice una formación que facilite su inserción en el mercado laboral bajo estándares de competitividad.

Siendo así, el cierre provisional de los centros educativos generaría consecuencias negativas a largo plazo entre las cuales estarían:

1. La baja en el número de niños y adolescentes vinculados a una institución educativa (escolarización) (MEN, 2020).

2. El aumento de niños, adolescentes y jóvenes estudiantes que abandonen sus procesos de formación (deserción escolar) (MEN, 2020). 
3. La baja en los índices de títulación formal en los diferentes niveles. Entonces, es evidente como a causa de la pandemia se profundizarán los desafíos que la educación, y particularmente la educación superior en Colombia, históricamente ha tenido que enfrentar.

A lo anterior se suma que, las universidades sufren riesgos sobre su sostenibilidad, flujo de caja y capacidad económica, lo que debilita su solidez institucional (Banco Interamericano de Desarrollo, BID, 2020). Estas proyecciones evidenciarían la difícil situación por la que atravesarían estudiantes, docentes, e instituciones de educación sino se generan acciones de amortiguamiento sobre las restricciones que se tienen para el desarrollo de la educación remota en el país.

A lo mencionado previamente se debe sumar la crisis económica local, nacional y mundial, pues se estima una afectación en este campo, en concreto del consumo privado y la inversión (Banco Mundial, 2021). En general, para un 77\% de las actividades de comercio, las ventas se redujeron entre un $80 \%$ y un $100 \%$, debido al cierre de establecimientos, bares, teatros, restaurantes y empresas textiles. Esto ha conducido a que durante la cuarentena el $44 \%$ de los comerciantes suspenda contratos laborales, otorgue licencias no remuneradas o envíe su personal a vacaciones (Semana, 2020). Cerca de un 47\% de los trabajadores colombianos se encuentra en la informalidad, siete de cada diez trabajadores no contribuyen al sistema de seguridad social, aspectos que resultan críticos respecto a la capacidad económica de la ciudadanía para afrontar la crisis (Rodríguez, 2020). Esta proyección, así como el generalizado y creciente ambiente de incertidumbre a nivel social y económico, se presenta como un factor que ocasiona estados de estrés, repercutiendo también en la capacidad de concentración y aprendizaje, así como en el bienestar físico y emocional no sólo de los estudiantes sino de toda la comunidad educativa.

Dicho esto, resulta pertinente comprender, tal como lo propone Bourdieu (1991), los efectos que la migración de la educación presencial a la remota, con ocasión de la pandemia del COVID-19, están provocando en las trayectorias personales y académicas de los estudiantes. Este es pues el objetivo de este escrito. De manera que este artículo se concentrará en tres dimensiones: el acceso a los recursos tecnológicos necesarios para la educación remota; las afectaciones en la salud mental; y, las afectaciones económicas y cambios en la vida familiar.

Es relevante elaborar esta indagación en todos los niveles de formación; empero, en este caso el análisis se centra en los estudiantes de una Institución de Educación Superior (IES), clasificada en el Sistema Nacional de Información de la Educación Superior - SNIES - , como una institución técnica profesional redefinida por ciclos propedéuticos. Dicha IES cuenta con cobertura a nivel nacional y ofrece una amplia variedad de programas, además, se caracteriza porque sus matriculados pertenecen principalmente a las clases populares de nuestro país.

\section{REVISIÓN DE LA LITERATURA}

Los costos económicos y psicológicos de la pandemia progresan conforme lo hacen las tasas de contagio y muertes por la enfermedad del COVID-19 a nivel mundial. Es por esto que, entre los efectos generalizados como producto de la pandemia, se ha reportado 
desesperanza sobre el futuro, síntomas de ansiedad y depresión (Boyraz \& Legros, 2020). Uno de los principales factores que ha contribuido a la angustia de las personas en los periodos de aislamiento ha sido la preocupación por el contagio de algún miembro de su núcleo familiar u otros seres queridos (CUNY Graduate School of Public Health and Health Policy, CUNY SPH, 2020). Por ejemplo, más de la mitad de las personas en China han percibido los impactos psicológicos de la pandemia como moderados o severos (Wang et al., 2020).

Además, siguiendo los resultados de encuestas realizadas en Bangladesh, otro país en vía de desarrollo, la mayoría de las personas cree que la pandemia pudo afectar negativamente su trabajo, ingresos o educación, y al menos la mitad de la población tendría alguna alteración en su salud mental (Banna et al., 2020). En conclusión, se sabe que las afectaciones en esta dimensión aumentan como consecuencia del aislamiento social, del miedo de resultar contagiado, de las pérdidas financieras y laborales, a lo que se suman los estresores monetarios como las dificultades de pago de la renta o la amenaza de embargos o desalojos.

A pesar de que este tipo de reacciones se pueden considerar naturales para una crisis de salud mundial, tanto hallazgos previos de Sprang \& Silman (2013) como evidencia reciente Boyraz y Legros (2020) sugieren que este tipo de experiencias pueden resultar traumáticas para algunas personas, lo que puede conllevar a un trastorno de estrés postraumático. Sprang y Silman (2013) sugieren que los estados de aislamiento pueden, en efecto, configurarse como una fuente de experiencias altamente traumatizantes para niños y adultos. Incluso, la evidencia recogida durante emergencias de salud anteriores, con altas tasas infecciosas y de mortalidad, sugiere que las personas con desventajas sociales, como aquellas con pocos ingresos, se verían afectadas de forma desproporcionada por la actual situación generada por el COVID-19 (CUNY SPH, 2020). Esto, pues a medida que aumenta la pobreza también se incrementa el riesgo de infectarse, ya que se tiene menor acceso a la higiene personal (Boyraz \& Legros, 2020). El Departamento de Salud Mental de Nueva York ha señalado la existencia de otros factores sociodemográficos que afectan la capacidad de las personas para adoptar medidas preventivas y de distanciamiento social. Por ejemplo, las personas en condición de desempleo, empleo familiar o de escasos recursos económicos tienden a permanecer menos en sus casas durante la semana laboral e implementar prácticas de distanciamiento tardíamente en comparación con las personas en posición de privilegio socioeconómico, quienes también se ven afectados por el virus, pero tienen mayor capacidad de protección y son el grupo con menor cantidad de casos de COVID-19 reportados (Valentino-DeVries, Lu \& Danza, 2020). De allí que, se puede decir que las brechas sociales conducen a una exposición desigual de riesgos entre las personas.

El apoyo social juega un rol protagónico para mantener la integridad psicológica, pero el distanciamiento social restringe la activación eficaz de esta estrategia de afrontamiento (Polizzi, Lynn \& Perry, 2020). En contraste con otro tipo de desastres donde las personas se integran física y socialmente con propósitos comunes para reconstruir lo destruido, se reducen las posibilidades para tejer mutuamente espacios de bienestar, indispensables para la salud mental. A esto se suman las consecuencias particulares que se manifiestan de diferentes formas en distintos contextos de la sociedad, principalmente debido al 
impacto económico, lo que puede llevar a afecciones agudas y la cronificación de condiciones previas en la salud mental.

En este panorama resultan indiscutibles las diversas situaciones que pueden estar enfrentando los estudiantes de educación superior para desempeñar sus actividades de formación, no solo por el formato remoto sino por las implicaciones que conlleva el confinamiento causado por una pandemia global. Por esto, en este artículo no se puede desconocer el impacto que tiene la titulación en educación superior para jóvenes de clases populares, particularmente pensando en el contexto colombiano. Autores como García, Espinosa, Jiménez y Parra (2013) y Bourdieu (1991) aseguran que la democracia escolar supone la democracia social (García et al., 2013), lo cual resalta la importancia de la educación para la igualdad de oportunidades y la movilidad social ascendente.

Sin embargo, la educación en las sociedades capitalistas y con tendencias políticas y económicas neoliberales tiene grandes dificultades no sólo en términos del acceso sino de la posibilidad de otorgar oportunidades reales de éxito académico y laboral (García et al., 2013). Este es el caso de Colombia, donde los jóvenes rurales y de las clases marginadas tienen bajas posibilidades de acceder a la educación superior, tal como lo propone García et al. (2013) "el aprendizaje es para unos una conquista" (p. 19). Se estaría, entonces, ante un panorama de exclusión que sería reforzado por la implementación obligatoria de la educación remota.

\section{Metodología}

Esta investigación se desarrolla como un ejercicio empírico, clasificado como un estudio de tipo descriptivo mediante encuestas (Hernández, Fernández y Baptista, 2014). También se ubica como una indagación cualitativa y de carácter exploratoria (Hernández et al., 2014). Estos enfoques epistemológicos permiten una aproximación al objeto de estudio que describe de manera transversal una muestra no probabilística para identificar la situación de los estudiantes de una IES frente a la continuación, imprevista, de sus estudios en modalidad remota. En este sentido, la indagación girará en torno a las tres dimensiones mencionadas, que se profundizan a continuación.

1. Dificultades de acceso a los recursos tecnológicos necesarios para la educación remota. En esta categoría se abordará la posibilidad que tienen los estudiantes de contar con una herramienta tecnológica como lo es un computador, una tablet $u$ otro dispositivo móvil, en tanto herramienta principal para acceder a las clases y sus contenidos. Aquí también, se comprenderá el acceso a conectividad, como segundo elemento imprescindible en los modelos de educación remota o virtual.

2. Afectaciones a la salud mental. El Plan Multisectorial de Apoyo para el COVID-19 de la Organización de Naciones Unidas, los centros de control de enfermedades del gobierno de Estados Unidos y la Organización Mundial de la Salud (Boyraz \& Legros, 2020), defienden la importancia de reconocer síntomas de estrés resultantes de los aislamientos y de la misma enfermedad del COVID-19. En esta dimensión se identifican los impactos a la salud mental señalados por los participantes del estudio en los distintos instrumentos empleados. 
3. Afectaciones a nivel económico y cambios en la vida familiar. Como se ha esgrimido a lo largo del artículo, los efectos negativos de la pandemia redundan con fuerza en lo económico, a través de las pérdidas de empleos, cierre de micro y medianas empresas, y un aumento de la informalidad. Esta situación no es ajena a los estudiantes y sus familias y, a largo plazo, impactará no solo su rendimiento académico sino las posibilidades de continuar con su proceso de formación educativa, esto es lo que se quiere abordar en esta dimensión. Y, en relación a los cambios en la vida familiar, se busca conocer como los encierros obligatorios y la convivencia permanente tienen efectos, positivos o negativos, sobre el estudiante.

Para lograr este abordaje se decidió por un diseño metodológico de tipo mixto pues articula técnicas cuantitativas y cualitativas (Creswell, 2015). Los datos cuantitativos, por su parte, fueron registrados mediante una encuesta de percepción, siendo esta una técnica adecuada de observación indirecta, aplicación masiva y estándar, para obtener datos mediante la interrogación a los participantes (García, 1993; Sierra, 1994). Y, los datos cualitativos, fueron recolectados mediante las técnicas de entrevista abierta y grupos focales; su objetivo fue captar a partir del encuentro y el discurso las distintas opiniones que circulan en un grupo seleccionado. Con la entrevista grupal, de un lado, se propuso comprender a profundidad los relatos, esto, entendiendo que en este tipo de entrevista "el grupo es un marco para captar las representaciones ideológicas, los valores o el imaginario dominantes en un determinado colectivo" (Valles, 1999, p. 286). De otro lado, con el grupo focal se propendió por "generar un entendimiento profundo de las experiencias y creencias de los participantes" (Mella, 2000, p. 24), en este caso sobre las tres dimensiones abordadas.

En relación con la validez de los instrumentos debe decirse que estos se crearon, al igual que este artículo, en el marco de un proceso de investigación mayor denominado "Plataforma virtual de servicios de acompañamiento y orientación para estudiantes, emprendedores y potenciales emprendedores que siguen Pa'lante en tiempos difíciles (2020-201)". Así pues, el contenido de los instrumentos se elaboró desde el marco teórico de este proyecto y desde un análisis contextual y de la literatura sobre los efectos que genera una pandemia en las poblaciones humanas, sumado a la investigación previa realizada sobre el universo de afectaciones que suelen sufrir las personas en escenarios similares de crisis.

En lo concerniente a la población de estudio, debe precisarse que en el caso de las encuestas y los grupos focales, los instrumentos se aplicaron a estudiantes de la IES, que cursan diferentes programas académicos y que se encuentran en momentos distintos de su proceso de formación. Sin embargo, para complementar este abordaje, se aplicó un instrumento adaptado a docentes y personal administrativo, pues desde sus diferentes roles son testigos y receptores de las percepciones y experiencias de sus estudiantes en la adaptación de la modalidad remota. Los participantes se incluyeron en el grupo de estudio mediante un muestreo no probabilístico por conveniencia.

Las encuestas, por un lado, se realizaron vía Google forms y su respuesta fue de carácter voluntario, así pues se obtuvo $N=1397$ respuestas desde los estudiantes, $N=219$ de docentes, y $N=17$ de personal administrativo. Entre la participación de 
los estudiantes, los programas académicos de los que hicieron parte fueron Administración de empresas $N=532$, Administración de empresas agroindustriales $N=20$, Administración de servicios de salud $N=122$, Administración turística y hotelera $N=81$, Comunicación social $N=56$, Contaduría pública $N=150$, Dirección y producción de medios audiovisuales $N=57$, Diseño de modas $N=29$, Diseño gráfico $N=110$, Ingeniería de sistemas $N=91$, Ingeniería electrónica $N=20$, Negocios internacionales $N=128$, y Publicidad y mercadeo $N=1$. Dentro del grupo de docentes, participaron voluntarios de las áreas Escuela de Ciencias Administrativas (ECA) $N=85$, Escuela de Comunicación y Bellas Artes (ECBA) $N=1$, Escuela de negocios $N=53$ y Escuela de ingeniería $N=80$. El grupo de administrativos fue conformado por los directores voluntarios pertenecientes a $\operatorname{ECA} N=11$, ECBA $N=2$, Escuela de negocios $N=2$ y Escuela de ingeniería $N=2$.

Por otro lado, la entrevista grupal y los grupos focales se realizaron por medio de videoconferencias y la participación fue voluntaria. La entrevista abierta tuvo como único foco a los estudiantes logrando una participación de 31 matriculados. En cuanto a los grupos focales, estos fueron dirigidos a los docentes y el personal administrativo. Siguiendo los requerimientos de esta técnica en la sesión de docentes se contó con la presencia de 10 profesores, y en la de administrativos con nueve asistentes.

Ahora pues, sobre la sistematización de la información en el caso de los grupos focales y las entrevistas se tomaron como ejes analíticos las tres dimensiones a abordar y su análisis. Continuando el proceso, la generación de resultados se dio siguiendo los parámetros de sistematicidad y rigurosidad, así como de interpretación propios de los métodos cualitativos (Ragin, 2007; Létorneau, 2009). Por su lado, los datos recogidos por las encuestas fueron analizados para obtener resultados descriptivos. Por tanto, se realizó conteo de frecuencias sobre las dificultades enfrentadas por los estudiantes durante la pandemia en las tres dimensiones analizadas, desde la perspectiva de docentes, administrativos, y los mismos estudiantes.

\section{Resultados}

—El problema es el internet, es el computador, es todo-.

\section{Resultados cualitativos de las dificultades de acceso} a los recursos tecnológicos para la educación remota

La entrevista abierta con los estudiantes, así como los grupos focales con docentes y personal administrativo, posicionó como una preocupación relevante las dificultades de acceso que tenían los estudiantes a una conexión de internet estable o a un dispositivo con las características adecuadas que les permitiera desarrollar con satisfacción sus clases. En este sentido, el empleo de las Tecnologías de la Información y la Comunicación (TIC) en la educación, principalmente en los modelos virtuales y a distancia, busca posibilitar una mayor fluidez en las comunicaciones entre las personas, fragmentando barreras, ubicadas en espacio y tiempo (Castellanos y Castro, 2018). 
Es así como en condiciones ideales las TIC potencializan la educación, favorecen la cobertura y la calidad al permitir acceder remotamente a recursos ilimitados. Sin embargo, ya se había mencionado en este artículo que dichas condiciones no están dadas para buena parte de los estudiantes de este país y esta indagación hizo evidente tal situación. Por un lado, se hicieron visibles las dificultades sobre el acceso a una red de internet con servicio constante.

Esto se evidencia en la afirmación realizada por uno de los docentes en el grupo focal, para quien:

Se sufre mucho por el tema del internet, sobre todo porque las clases son en hora pico y todo el mundo está conectado y eso genera bastante intermitencia en la conectividad. Uno ve chicos que se desconectan cada rato de la sesión y solo dicen — profe es que se me cayó el internet—, para verle el lado bueno los molesto diciendo que se van a tomar sopa.

Esta no es una preocupación nueva, ni a nivel nacional ni regional, al respecto mencionan García y García (2020) que en la mayor parte de las políticas digitales en educación de la región no se priorizó el abordaje de la cobertura y calidad de internet, razón por la cual el desarrollo de las actividades académicas se han visto fuertemente afectadas.

Aunque los problemas de conectividad se postulaban como un reclamo general, también se hizo evidente como este problema se agravaba en la ruralidad. Al respecto, un director de programa aseguró:

La dificultad primordial es el tema de la conectividad, si yo hablo por ejemplo de Ipiales, las personas que estudian los fines de semana, en su mayoría son de municipios y veredas, entonces para ellos es muy complejo, es bastante traumático el cambio a la mediación virtual en el tema de las clases.

Este asunto no es propio de la IES analizada, Acero, Briceño, Orduz y Tuay (2021) muestran como esta realidad que se caracteriza por las carencias en la infraestructura tecnológica y de conectividad se replica a lo largo del país. Por su parte, Gutierrez y Morales (2020), revelaron un panorama similar en su investigación sobre el sistema educativo peruano.

En la entrevista grupal los estudiantes manifestaron que este problema también ocurría en las urbes. Esto comentó uno de los jóvenes:

Yo entiendo que estamos en lo virtual por el tema de la pandemia que es nacional, bueno más bien es mundial, no es un tema caprichoso de la U, porque a todos nos está tocando igual, hasta mi hermanito del colegio, pero es muy duro que se tengan que parar tantas veces las clases porque a los compañeros se les cae el internet y a los profes les toca repetir y así no se avanza. Aunque yo entiendo, bueno unos son por vagos que dicen que se les desconecta el internet, hay otros que están en otras partes y allá el internet es malo, si hasta a mi me falla y yo estoy en Bogotá.

Sobre esto tema otro de los estudiantes comentó: - Yo vivo en Soacha y tengo muchos problemas de internet, y eso que tengo contratado en la casa el servicio pero cuando se cae es un problema que vengan hasta acá a arreglarlo y tengo compañeros que les pasa lo mismo y viven en Bogotá, es que acá nos toca duro-. Estos resultados demuestran una falencia estructural en la implementación de la educación remota en el país y la imperiosa 
necesidad de entregar herramientas a las IES por parte del gobierno nacional para sortear este obstáculo, así como la necesidad de la emergencia de proyectos de innovación en las instituciones que, conscientes de su contexto y del de sus estudiantes, genere alternativas viables para su formación en un ambiente no presencial.

—Es que así es difícil estudiar—.

\section{Resultados cualitativos de las afectaciones a la salud mental}

Las afectaciones físicas y mentales que la pandemia ha generado han sido evidentes y se han reflejado en el desempeño académico de los estudiantes (Vanegas, 2020), este asunto fue descrito por una de las jovenes quien aseguró que "con el miedo a contagiarse y estar acá encerrada todo el tiempo pues uno le pierde la motivación al estudio". Se entiende con esta cita cómo el estrés, el aburrimiento, la angustia y la ansiedad aparecen en la vida de los estudiantes. Valdivieso et al. (2020), realizaron una pesquisa específica al respecto y concluyeron que "los estudiantes consideran al estado de ánimo durante la cuarentena un factor que afectó de manera negativa y fuerte la adquisición de nuevos conocimientos" (p. 279). En relación con la propuesta de estos autores, los hallazgos que se describen en este ítem se encaminan hacia el mismo argumento. De hecho, los grupos focales demostraron cómo los docentes se percataron pronto de esta situación y decidieron establecer estrategias para estimular e incentivar a los jóvenes: "Uno los entiende (a los estudiantes) pero les trata de buscar el lado bueno, generando actividades nuevas o abriendo espacios en las clases para que se expresen”, mencionó un docente.

Los resultados de los instrumentos cualitativos demostraron que para los estudiantes la participación en las clases presenciales, y en general las dinámicas que conlleva la enseñanza presencial, eran considerados espacios de autonomía, que se perdieron al verse obligados a recibir todas las clases en modalidad remota. Esto desató para muchos un sentimiento de frustración que sumado a la obligación de permanecer en su casa con pocas o nulas posibilidades de salidas, estaba detonando una serie de sentimientos negativos que afectaban al estudiante en sus diferentes dimensiones.

— Si hay para el arriendo no hay para la matrícula—.

\section{Resultados cualitativos de las afectaciones a nivel económico y cambios en la vida familiar}

Las consecuencias económicas de la pandemia afectaron de manera desigual a los estudiantes de las IES, para los más vulnerables esto les significó un cambio de vida o la pérdida de ciertos accesos a recursos y servicios que habían logrado gracias a sus ingresos económicos, que muchas veces provenían de la informalidad. Uno de los directivos de la IES donde se realizó estudio mencionó al respecto

Acá la mayoría de estudiantes estudia y trabajan pero están vinculados al mercado laboral desde la informalidad y las ganancias son mínimas. Por eso a ellos se les dificulta tener un ahorro con el que puedan soportar en este momento su casa, sus necesidades y sus obligaciones. 
En el caso analizado se hizo evidente la inquietud por parte de los directivos y los docentes por las altas probabilidades que existían de que los estudiantes no continuaran con sus estudios por motivos económicos. Desde la perspectiva de los jovenes las preocupaciones en términos económicos se dirigian especialemente en dos direcciones. Por un lado, la falta de recursos para la solvencia de temas económicos básicos, por lo cual suele sacrificarse el monto dedicado a la formación académica. Este asunto puede verse reflejado en una frase pronunciada por uno de los estudiantes en la entrevista abierta: "con esta situación tan difícil, si hay para el arriendo no hay para la matrícula”. Por otro lado, aparecía la inquitud de los estudiantes que dependían de sus padres por la posibilidad de que estos quedaran desempleados y que la inversión que se hace en la matrícula luego sea requerida para temas esenciales. Una de las estudiantes aseguró, "uno siente incertidumbre porque en cualquier momento saquen a mis papás de donde están trabajando y luego de pagar la matrícula, qué tal yo pueda estudiar pero no tengamos con que comer, o pagar la luz”.

Esta situación no es una inquietud propia de esta institución ni de sus miembros. La Asociación Colombiana de Universidades (ASCUN, 2020) confirmó una disminución del 11.3\% en las matrículas para el segundo semestre del 2020 a nivel nacional. Avellaneda y Elizondo (2021), por su parte, aseguran que esta situación no es exclusiva de las IES, es más bien una realidad compartida por los estudiantes de colegio de las clases sociales menos favorecidas quienes se retiran para ingresar al mercado laboral o buscar otra salida que les permita aportar económicamente a su círculo familiar. Los resultados en este sentido demuestran que las afectaciones económicas generadas por la pandemia han obligado a los estudiantes a replantear la continuación de sus estudios e incluso su finalización a largo plazo.

De otro modo, en relación con los cambios familiares, los estudiantes llamaron la atención sobre la sobrecarga de tareas, particularmente para aquellos que eran padres. Con esto se referían específicamente a la demanda constante de atención, vigilancia y ayuda de parte de sus hijos, y la acumulación de las tareas del hogar, que se suman a las labores que desarrollan en un entorno laboral y a las clases y trabajos propios de su formación académica. Esta situación es descrita por una estudiante:

Con la pandemia las cosas cambiaron mucho, a mi, por ejemplo, me toca hacerme cargo de mis hijos, del colegio, de la casa, del trabajo y del estudio, y el tiempo no da para tanto".

Cervantes-González y Hernández (2020) aseguran que "la madre tuvo que ocupar su tiempo, además de las actividades que tradicionalmente realiza, a estar presente durante las clases en línea de sus hijos en casa" (p. 95).

Con esto, no se puede olvidar que la pandemia está obligando a que las personas reinventen su vida cotidiana, no sin antes generar alteraciones, positivas y negativas, sobre las rutinas y dinámicas familiares.

\section{Encuesta de dificultades y afectaciones enfrentadas por los estudiantes}

El cuestionario en el que participaron estudiantes, docentes y personal administrativo permitió distinguir entre estos grupos las proporciones que se consideraron respecto a las dificultades enfrentadas por los estudiantes durante la pandemia. Entre los estudiantes, las mayores dificultades o afectaciones a las que indicaron enfrentarse durante la 
pandemia son la falta de recursos para pagar la matrícula ocasionada por la pandemia (desempleo, cancelación o licencias no remuneradas de contratos) (64.6\%) y la priorización de gastos familiares (necesidad de invertir el dinero de la matrícula en otras cosas) (40.7\%) (Figura 2), mientras aquellos aspectos señalados en menor proporción resultaron ser los problemas familiares (4.2\%) (Figura 3), el acceso a un computador o dispositivo para conectarse a las clases y/o hacer trabajos (14.5\%) (Figura 1) y la falta de recursos para pagar la matrícula por razones ajenas a la pandemia (14.8\%) (Figura 2).

Para el caso de los docentes, las mayores proporciones se dieron alrededor de las dificultades o afectaciones de conectividad y acceso a internet (79.5\%) (Figura 1) y falta de recursos para pagar la matrícula ocasionada por la pandemia (73\%) (Figura 2), mientras los ítems señalados en menor proporción fueron problemas familiares (7.8\%) (Figura 3) y falta de recursos para pagar la matrícula por razones ajenas a la pandemia (17.3\%) (Figura 2); en comparativa, se observa que entre los docentes se percibe mayor proporción de dificultades o afectaciones que enfrentan los estudiantes, respecto a las proporciones percibidas entre los estudiantes.

En cuanto al personal administrativo, todas las dificultades o afectaciones se percibieron en mayor proporción respecto a los estudiantes y docentes, a excepción de la falta de recursos para pagar la matrícula por razones ajenas a la pandemia (11.7\%) (Figura 2) y la conectividad y acceso a internet (70.6\%) (Figura 1$)$.

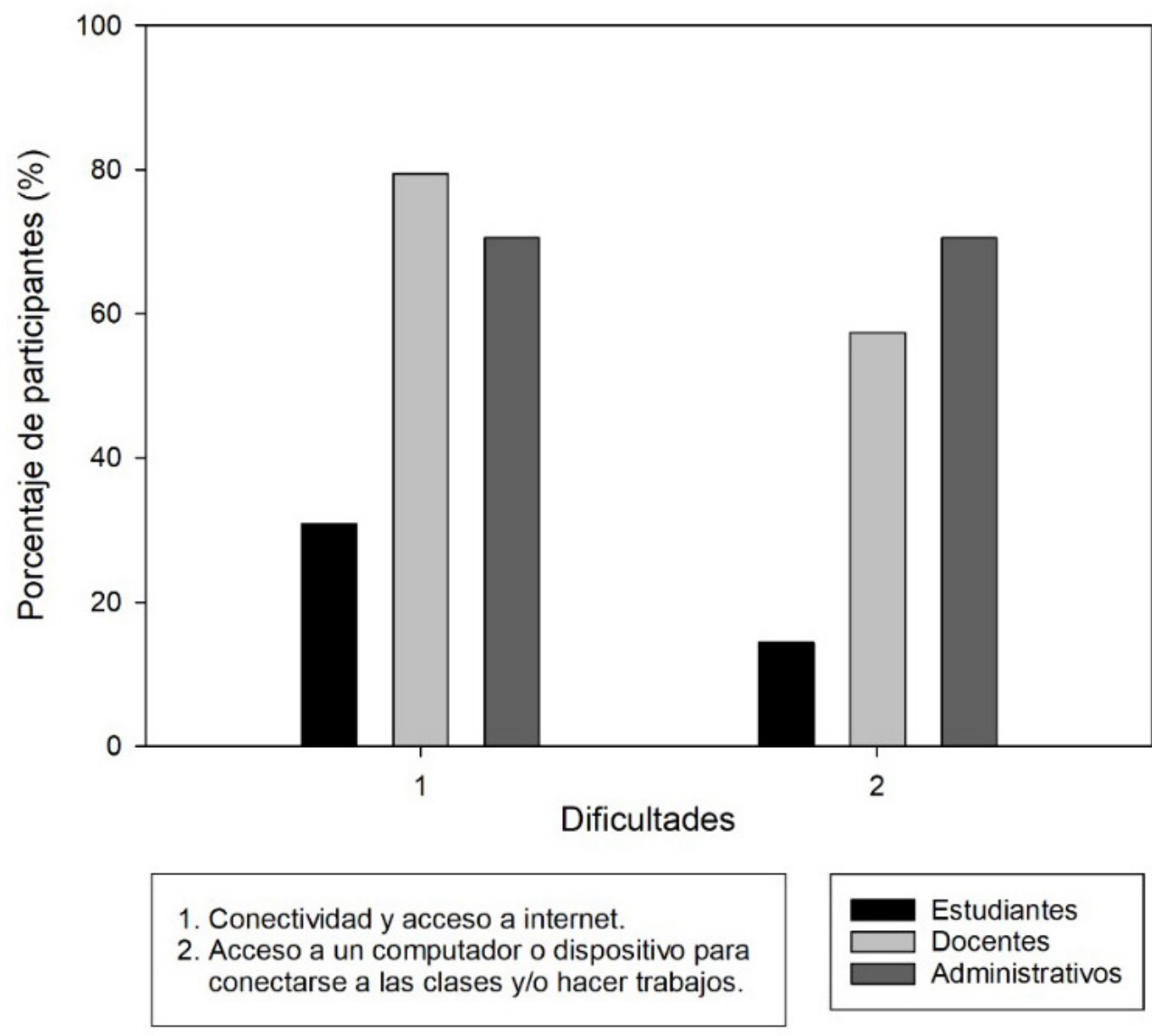

Figura 1. Dificultades de acceso a recursos tecnológicos que enfrentan los estudiantes durante la pandemia.

Nota: Proporciones calculadas para las dificultades percibidas por los participantes. Fuente: Elaboración propia. 


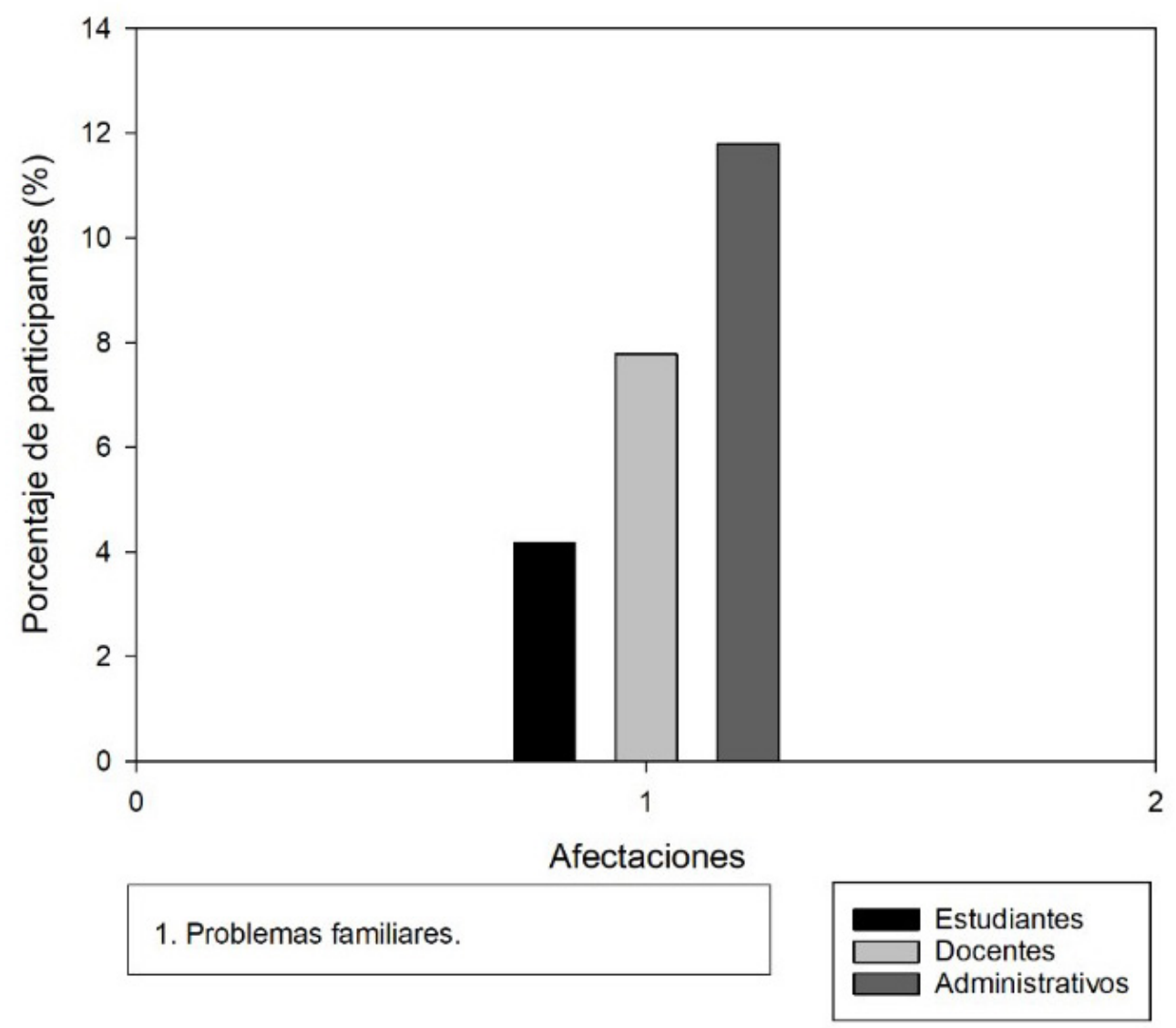

Figura 2. Afectaciones de salud mental y física que enfrentan los estudiantes durante la pandemia. Nota: Proporciones calculadas para las dificultades percibidas por los participantes.

Fuente: Elaboración propia.

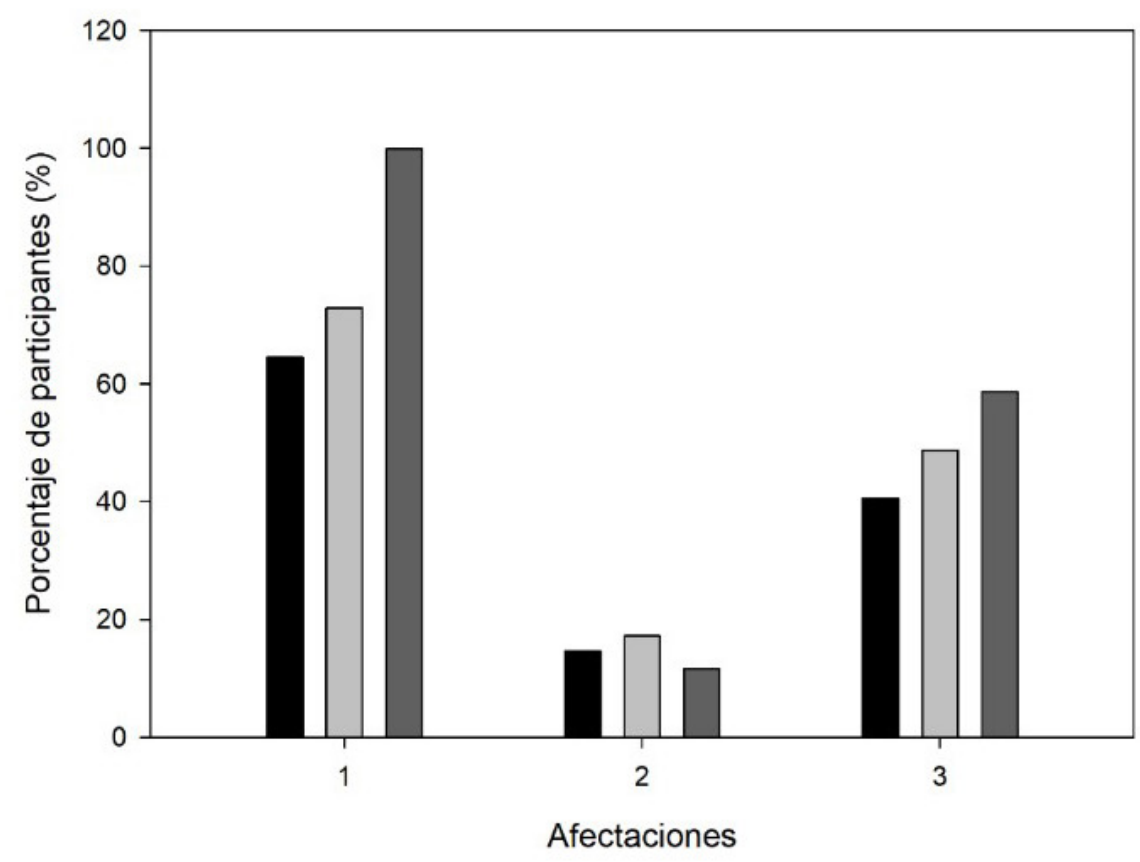

1. Falta de recursos para pagar la matrícula ocasionada por la pandemia (desempleo, cancelación o licencias no remuneradas de contratos).

2. Falta de recursos para pagar la matrícula por razones ajenas a la pandemia.

conectarse a las clases y/o hacer trabajos.

3. Priorización de gastos familiares (necesidad de invertir el dinero de la matrícula en otras cosas).

Figura 3. Afectaciones económicas y cambios en la vida familiar que enfrentarn los estudiantes durante la pandemia.

Nota: Proporciones calculadas para las dificultades percibidas por los participantes.

Fuente: Elaboración propia. 
Siguiendo estos resultados es posible afirmar que, contrario a lo esperado, las dificultades de acceso a recursos tecnológicos por parte de los estudiantes no fueron consideradas excesivas (mas no son despreciables) para los mismos aun cuando se ha documentado una amplia desigualdad en esta distribución de acceso (CEPAL, 2020) y se ha resaltado el hecho de que la población estudiantil de la IES pertenece a estratos socioeconómicos bajo y medio. Igualmente, para las afectaciones de salud mental y física que enfrentan los estudiantes en pandemia, como los problemas familiares, se esperaba una proporción mayor de estudiantes afectados, situación que sí es sobreestimada por docentes y personal administrativo.

Esto debido a que, en general, se ha reportado previamente un aumento significativo del estrés familiar derivado de estados de alerta en la salud pública por otras epidemias infecciosas (Boyraz \& Legros, 2020), pero apenas un 4.2\% de los estudiantes señalaron este tipo de afectación. Sin embargo, para las afectaciones económicas y cambios en la vida familiar que enfrentan los estudiantes, las proporciones señaladas por los tres grupos de participantes respecto a la falta de recursos económicos para pagar la matrícula ocasionada por la pandemia y la priorización de gastos familiares, resultan consistentes con la situación de crisis, las consecuencias y el deterioro del sistema económico a nivel nacional y global, por lo que era esperable que estas proporciones resultaran particularmente altas.

\section{Conclusiones}

El cambio inesperado e improvisado de la presencialidad a la educación remota le dio un giro total a la educación superior en Colombia. Los resultados demostraron que la manera apresurada en que se implementó esta modalidad de educación dio por sentado que los estudiantes gozaban de recursos materiales, tecnológicos, y servicios fundamentales para desarrollar las clases en modalidad remota. Con el paso de las semanas, la ampliación de las fechas y la aplicación de las medidas restrictivas y los confinamientos se hicieron evidentes los obstáculos que existían para una aplicación unificada de esta modalidad en la educación superior.

Los resultados en términos de problemas de conectividad, incluso de imposibilidad de acceder a una red de internet, hacen evidentes las demandas que se tienen frente al gobierno para llevar este servicio, que en la actualidad se considera básico, a todas las regiones del país, principalmente a los territorios rurales alejados. Lograr importantes avances en este frente permitiría ampliar las tasas de cobertura educativa a nivel nacional, pues la educación virtual se volvería no solo una alternativa sino una realidad para muchos jóvenes que no cuentan con los recursos para desplazarse a una ciudad central. Pero, a su vez, hace un llamado a las IES, y en general, a todas las entidades de educación, a presentar nuevas alternativas de formación por medio de proyectos de innovación pedagógica que acerquen a las personas, más allá de todas sus limitaciones a la educación.

Por su parte, los efectos en la salud mental no fueron representados de forma proporcional por las técnicas utilizadas. Es decir, si bien en las técnicas cualitativas fueron reconocidos distintos estresores mencionados en los antecedentes del estudio, en la observación cuantitativa fue señalado por una minoría de estudiantes la ocurrencia de problemas familiares que hayan tenido que enfrentar como parte de los cambios en la salud mental. Sin embargo, 
como se evidenció en los grupos focales de acuerdo a los estudiantes, las dificultades económicas como la falta de recursos ocasionada por la pandemia y la priorización de gastos familiares, que resultaron ser las afectaciones que se presentaron con mayor intensidad, en efecto se sumarían a aquellas situaciones que se configuran como factores de estrés en la vida de los estudiantes. De manera que, esta indagación demostró como la pandemia al profundizar las brechas sociales y complejizar el acceso y permanencia de las poblaciones vulnerables a la educación superior, también activa una carga emocional negativa relacionada con el estrés que genera la escasez de recursos.

Este artículo abre el espacio para que se realicen indagaciones similares colocando en el centro las percepciones y experiencias de docentes y directivos en el marco de la educación remota. Así como el análisis de las mismas dimensiones aquí tratadas en estudiantes de básica y secundaria.

\section{REFERENCIAS}

Acero, O., Briceño, A., Orduz, M. y Tuay, R. (2021). Realidades de la educacion rural en Colombia, en tiempos de covid- 19. [Investigación]. https://doi.org/10.15332/ dt.inv.2021.02415

Amat, Y. (septiembre 12, 2002). Cada día hay 249 denuncias por violencia intrafamiliar en Colombia. eltiempo. [online]. Disponible en https://www.eltiempo.com/justicia/ investigacion/fiscalia-cada-dia-hay-249-denuncias-por-violencia-intrafamiliar-en-colombia-537550

ASCUN. (2020). ASCUN revela que matrículas del año 2020 disminuyeron, aunque no tanto como se proyectaba. [Online]. Disponible en https:/www.ascun.org.co/noticias/ detalle/ascun-revela-que-matriculas-del-ano-2020-disminuyeron-aunque-no-tanto-como-se-proyectaba

Avellaneda, D. y Elizondo, N. (2021). Implementación de políticas educativas. En, J. Neri, R. Medina, M. Medina y P. González, Efectos sociales, económicos y de la salud ocasionados por la pandemia del COVID19. México, D.F.: Plaza y Valdés.

BID. (mayo, 2020). La educación superior en tiempos de COVID-19: Aportes de la Segunda Reunión del Diálogo Virtual con Rectores de Universidades Líderes de América Latina. New York. BID/universia/Santander. Recuperado de https://publications.iadb. org/publications/spanish/document/La-educacion-superior-en-tiempos-de-COVID19-Aportes-de-la-Segunda-Reunion-del-Di\%C3\%A1logo-Virtual-con-Rectores-deUniversidades-Lideres-de-America-Latina.pdf

Banco Mundial. (March 24, 2021). Colombia: panorama general. [Online]. Disponible en https://www.bancomundial.org/es/country/colombia/overview\#1

Banna, M. H. A., Sayeed, A., Kundu, S., Christopher, E., Hasan, M. T., Begum, M. R., Kormoker, T., Dola, S. T. I., Hassan, M. M., Chowdhury, S. \& Khan, M. S. I. (2020). The impact of the COVID-19 pandemic on the mental health of the adult population in Bangladesh: a nationwide cross-sectional study. International Journal of Environmental Health Research, 1-12. https://doi.org/10.1080/09603123.2020.1802409 
Bourdieu, P. (1991). El sentido práctico. Madrid: Taurus.

Boyraz, G. \& Legros, D. N. (2020). Traumatic Stress: Probable Risk Factors and Correlates of Posttraumatic Stress Disorder. Journal of Loss and Trauma, 25(6-7), 503-5222. https://doi.org/10.1080/15325024.2020.1763556

Castellanos, E. y Castro, J. (2018). Aproximación Teórica para el uso de los Entornos Virtuales en el Proceso de Aprendizaje de los Estudiantes Universitarios. Revista Scientific, 3(7), 99-120. https://doi.org/10.29394/Scientific.issn.2542-2987.2018.3.7.5.99-120

CEPAL. (2020). La educación en tiempos de la pandemia del COVID-19. Santiago: ONU. Recuperado de https://repositorio.cepal.org/bitstream/handle/11362/45904/S2000510_ es.pdf? sequence $=1 \&$ is Allowed $=y$

Cervantes-González, E. y Hernández, M. (2020). El emprendimiento de padres de familia con la modalidad de clases en línea durante la pandemia del COVID-19. Emprennova, 1(2), 86-112. Disponible en http://emprennova.uaq.mx/index.php/ojs/article/view/21

Creswell, J. W. (2015). A concise introduction to mixed methods research. Ann Arbor: Sage.

CUNY SPH. (2020). Week 4. [CUNY New York City COVID-19 Survey Week 4]. Available: https://sph.cuny.edu/research/covid-19-tracking-survey/week-4/

García, M. (1993). La encuesta. En, M. Garcia, J. Ibáñez y F. Alvira, El análisis de la realidad social. Métodos y técnicas de Investigación [pp. 141-170]. Madrid: Alianza Universidad Textos.

García, J. y García, S. (Comp.) (2020). Las tecnologías en (y para) la educación. Montevideo: FLACSO. Recuperado de https://www.flacso.edu.uy/publicaciones/edutic2020/ garcia_garcia_tecnologias_en_y_para_la_educacion.pdf

García, M., Espinosa, J., Jiménez, F. y Parra, J. D. (2013). Separados y desiguales educación y clases sociales en Colombia. Bogotá, D.C.: Dejusticia. Disponible en https:// www.dejusticia.org/publication/separados-y-desiguales-educacion-y-clases-socialesen-colombia/

Gutiérrez, Z. y Morales, I. (2020). Una mirada a la política educativa. Revista Dilemas Contemporáneos: Educación, Política y Valores, 2(84), 98-99. https://doi.org/10.46377/ dilemas.v32i1.2040

Hernández, R., Fernández, C. y Baptista, P. (2014). Metodología de la investigación (6 ed.). México, D.F.: Mc Graw Hill.

Létourneau, J. (2009). La caja de herramientas del joven investigador. Medellín: La Carreta Editores.

Mella, O. (2000). Grupos focales. Técnica de investigación cualitativa. [Docuemento de Trabajo No. 3]. Santiago de Chile: CIDE. Recuperado de http://files.palenque-de-egoya.webnode.es/200000285-01b8502a79/Grupos\%20Focales\%20de\%20Investigaci\%C3\%B3n. $\operatorname{pdf}$

Palacios, N. (2020). Aproximaciones académicas de estudiantes y profesores de la Facultad de Educación de la Universidad de los Andes. [Online]. Disponible en https://uniandes. edu.co/es/noticias/educacion/educacion-y-covid19-aproximaciones-desde-la-academia 
Polizzi, C., Lynn, S. J. \& Perry, A. (2020). Stress and coping in the time of COVID-19: Pathways to resilience and recovery. Clinical Neuropsychiatry, 17(2), 59-62. https:// doi.org/10.36131/CN20200204

Radinger, T., Echazarra, A., Guerrero, G. y Valenzuela, J. (2018). OCDE Revisión de Recursos Escolares COLOMBIA. [Resumen]. Recuperado de www.oecd.org/education/ schoolresourcesreview.htmwww.oecd-ilibrary.org

Ragin, Ch. (2007). La construcción de la investigación social: introducción a los métodos y su diversidad. Bogotá, D.C.: Siglo del Hombre Editores.

República de Colombia. DANE. (2021). Caracterización pobreza monetaria y resultados clases sociales 2020. [Presentación]. Recuperado de https://www.dane.gov.co/files/investigaciones/condiciones_vida/pobreza/2020/Presentacion-pobreza-monetaria-caracterizacion-clases-sociales-2020.pdf

República de Colombia. DANE. (2019). Indicadores básicos de tenencia y uso de Tecnologías de la Información y Comunicación - TIC en hogares y personas de 5 y más años de edad. [Boletín Técnico]. Bogotá, D.C.: DANE. Recuperado de https://www.dane.gov. co/files/investigaciones/boletines/tic/bol_tic_hogares_departamental_2018.pdf

República de Colombia. MEN. (October 25, 2020). Trabajamos en equipo por prevenir y mitigar los impactos del COVID- 19 en la deserción en educación Preescolar, Básica, Media y Superior. [Online]. Disponible en https://www.mineducacion.gov.co/1759/w3article-401634.html?_noredirect=1

Rodríguez, E. (2020). Colombia impacto económico, social y político de la COVID-19. Análisis Carolina, (24), 1-14. Recuperado de https://www.fundacioncarolina.es/wp-content/ uploads/2020/04/AC-24.-2020.pdf

Semana. (April 30, 2020). La crisis económica que atraviesa la clase media en Colombia. Semana. [Online]. Disponible en https://www.semana.com/semana-tv/el-poder/articulo/covid-19-la-crisis-economica-que-atraviesa-la-clase-media-en-colombia/667210/

Sierra, R. (1994). Técnicas de Investigación social. Madrid: Paraninfo.

Sprang, G. \& Silman, M. (2013). Posttraumatic stress disorder in parents and youth after health-related disasters. Disaster Medicine and Public Health Preparedness, 7(1), 105-110. https://doi.org/10.1017/dmp.2013.22

UNESCO/IESALC. (2020). COVID-19 y educación superior. De los efectos inmediatos al día después. [Documentos de Trabajo]. Recuperado de https://www.iesalc.unesco.org/ wp-content/uploads/2020/04/COVID-19-070420-ES-2-1.pdf

Valdivieso, M., Burbano, V. y Burbano, Á. (2020). Percepción de estudiantes universitarios colombianos sobre el efecto del confinamiento por el coronavirus, y su rendimiento académico. Revista Espacios, 41, 269-281. https://doi.org/10.48082/espacios-a20v41n42p23

Valentino-DeVries, J., Lu, D. \& Danza, G. (April 3, 2020). Location Data Says It All: Staying at Home During Coronavirus Is a Luxury. The New York Times. [Online]. Available: https://www.nytimes.com/interactive/2020/04/03/us/coronavirus-stay-home-richpoor.html 
Valles, M. S. (1999). Técnicas de conversación y narración III. Los grupos de discusión y otras técnicas afines. In: S. Valles, Técnicas cualitativas de invesitgación social (Vol. 1, pp. 279-334). Madrid: Síntesis.

Vanegas, O. (2020). La motivación y planificación como reto pedagógico para el desarrollo del aprendizaje autónomo, que deben asumir los docentes con estudiantes de educación superior presencial, en transición a educación remota asistida por las TIC en Colombia en contexto de pandemia. [Monografia]. UNAD, Ibague, Colombia. Recuperado de https://repository.unad.edu.co/bitstream/handle/10596/38029/olvanegasv. pdf? sequence $=1 \&$ isAllowed $=y$

Wang, C., Pan, R., Wan, X., Tan, Y., Xu, L., McIntyre, R. S., Choo, F. N., Tran, B., Ho, R., Sharma, V. K. \& Ho, C. (2020). A longitudinal study on the mental health of general population during the COVID-19 epidemic in China. Brain, Behavior, and Immunity, 87, 40-48. https://doi.org/10.1016/j.bbi.2020.04.028

Andrea Katherine García González es Magíster en Estudios Sociales y socióloga de la Universidad del Rosario. Intereses de investigación en estudios sobre emprendimiento y empresarialidad, la enseñanza en emprendimiento, la sociología de la educación, la innovación pedagógica, investigadora y líder del grupo de investigación ESCALA (Categoría C en Minciencias) de la Corporación Unificada Nacional de Educación Superior CUN. https:// orcid.org/0000-0003-4589-9753

Danilo Esteban Rodríguez Zapata es Psicólogo con 3 años de experiencia en diseño, investigación y gestión de productos tecnológicos en formato AWP para la educación y el emprendimiento desde la psicometría. Analista estadístico y de desarrollo para validación de modelos de medición y operacionalización de constructos psicológicos y empresariales. https://orcid.org/0000-0003-0291-7050 